\title{
BMJ Open General practitioners' perceptions towards the use of digital health services for citizens in primary care: a qualitative interview study
}

\author{
Asbjørn Johansen Fagerlund, ${ }^{\odot}$ Inger Marie Holm, Paolo Zanaboni
}

To cite: Fagerlund AJ, Holm IM, Zanaboni P. General practitioners' perceptions towards the use of digital health services for citizens in primary care: a qualitative interview study. BMJ Open 2019;9:e028251. doi:10.1136/ bmjopen-2018-028251

- Prepublication history for this paper is available online. To view these files, please visit the journal online (http://dx.doi. org/10.1136/bmjopen-2018028251).

Received 30 November 2018 Revised 19 February 2019 Accepted 28 March 2019

Check for updates

(C) Author(s) (or their employer(s)) 2019. Re-use permitted under CC BY-NC. No commercial re-use. See rights and permissions. Published by BMJ.

Department for Patient Pathways, Norwegian Centre for E-health Research, Troms $\emptyset$, Norway

Correspondence to Asbjørn Johansen Fagerlund; asbjorn.johansen.fagerlund@ ehealthresearch.no

\section{ABSTRACT}

Objectives To explore general practitioners' (GPS) perceptions towards use of four digital health services for citizens: an electronic booking service to make reservations with the GP; an electronic prescription service to request renewal of maintenance drugs; a service for text-based non-clinical enquiries to the GP office and a service for text-based electronic consultation (e-consultation) with the GP.

Design A qualitative study based on semi-structured interviews.

Setting Primary care.

Participants Nine GPs who were early adopters of the four services were interviewed.

Method One moderator presented topics using openended questions, facilitated the discussion and followed up with further questions. Phone interviews were conducted, audio recorded and transcribed verbatim. Qualitative data were analysed using the framework method.

Results The use of digital services in primary care in Norway is growing, although the use of text-based e-consultations is still limited. Most GPs were positive about all four services, but there was still some scepticism regarding their effects. Advantages for GP offices included reduced phone load, increased efficiency, released time for medical assessments, less crowded waiting rooms and more precise communication. Benefits for patients were increased flexibility, autonomy and time and money savings. Children, the elderly and people with low computer literacy might still need traditional alternatives. Conclusions More defined and standardised routines, as well as more evidence of the effects, are necessary for large-scale adoption.

\section{INTRODUCTION}

All general practitioners (GPs) participating in this study are private practitioners who have established a contractual agreement with the municipality in which their practice serves the population. They are financed through a variety of grants and reimbursements from the public sector, as well as out-ofpocket payments. ${ }^{1}$ The organisation of GPs in the Norwegian public healthcare system is referred to as the 'general practitioners
Strengths and limitations of this study

The study investigates technology implementation in general practice.

- Rigorous application of qualitative framework theory.

- The results highlight themes that are relevant for clinicians and policy-makers.

- All general practitioners (GPs) who participated in the study were voluntary early adopters, and thus possibly more positive and competent with technology than the average GP population.

scheme'. In an effort to provide citizens with a uniform portal for communicating with their GP, the Norwegian Directorate for e-health has developed the 'Digital dialogue with the general practitioner', a suite of four online services. By July 2018, these services were offered by 186 GP offices, which were part of the public GP scheme (4732 GPs working in 1542 offices), in order to obtain user experiences prior to large-scale deployment. The four digital services are accessible to citizens from the private section of the national portal helsenorge.no. They are available after login and include the following:

1. An electronic booking service to make reservations with the GP.

2. An electronic prescription service to request renewal of maintenance drugs, with direct integration with the electronic prescription system of pharmacies.

3. A service for text-based, non-clinical enquiries to the GP office (eg, opening hours, results from diagnostic tests).

4. A service for electronic consultation (e-consultation) with the GP.

The first three services are mainly administrative, geared towards introducing less time-consuming routines for both GP offices and patients, and free of charge for patients. E-consultation is a clinical service, which requires patients to pay the same 
out-of-pocket fee as for office visits. GPs are free to offer patients all four of the services or only some of them. The portion of the population with Internet access in Norway is very high, and almost total in the younger part of the population. ${ }^{2}$ It is therefore possible to assume that these electronic services have a large potential user base.

Being the only clinical service in the suite, the e-consultation service warrants a more detailed description. The service is a text-based service that is available on the same web page as the rest of the services. The patients initiate an e-consultation by logging into a level 4 security portal, where a written message can be sent to the GP. The message arrives in the GP's electronic patient record system, and can then be answered. The service currently offered in this study conforms to the definition that e-consultation is an asynchronous, non-face-to-face consultation using a secure electronic communication platform, ${ }^{3}$ where the doctor answers clinical questions in a similar way to a standard consultation and answers are sent electronically. ${ }^{4}$ The demand for services that enable digitally based communication between the patients and their GPs is not new and has been around since the early days of the Internet. ${ }^{5}$ While the use of online services in healthcare is on the rise, it is unclear whether it benefits all socioeconomic segments of the population. ${ }^{6}$ E-consultations in primary care have been mostly used to increase access to specialist care.$^{7-10}$ However, the proliferation of e-consultations and other digital health services used by citizens to communicate directly with their GPs is limited, and consequently the body of literature from which to draw direct comparisons is narrow.

The presented suite of digital health services for citizens has the potential to improve the accessibility and efficiency of primary healthcare. However, government and vendors have been criticised for being overly optimistic about the expected favourable outcomes from employing health informatics. ${ }^{11}$ In order to release some of the potential of health informatics systems, it has been argued that, among others, feature functionality, project management and user-related outcomes affect implementation outcomes. ${ }^{12}$ The aim of this study was to explore GPs' perceptions towards the use of digital health services for citizens in primary care. Three main research questions were addressed: (1) Which routines were implemented by GPs who adopted digital health services for citizens? (2) What were GPs' impressions of benefits and disadvantages of digital health services for citizens? (3) How did GPs use digital health services for citizens?

\section{METHODS}

\section{Patient and public involvement}

This study did not have patient involvement. All participants were voluntary medical professionals (GPs). The results will be distributed to the study participants via e-mail mailing lists that include both study participants and other GPs who used the four services.

\section{Ethics}

According to the Norwegian Act on Medical and Health Research $\$ 2$ and $\S 4$, the study did not require approval from the regional ethics committee (REK), but the procedure for handling the data was approved by the Data Protection Officer of the University Hospital of North Norway.

\section{Data collection}

We conducted semi-structured interviews with GPs who adopted at least one of the four digital services. Some GPs adopted the services since their early introduction in 2015, some started using the services in 2016, while others adopted them only a few months before the present study was conducted. We aimed to include 8-12 GPs, and conducted interviews until we observed that interviewees began to repeat themes. After nine interviews, we decided that the additional insight gained from each additional interview was diminishing, and concluded that data saturation had been reached ${ }^{13}$ for the main analytical categories.

A semi-structured interview guide was used to illustrate GPs' perceptions towards use of digital health services for citizens. The interview guide was developed with a number of questions for each of the four digital health services, as well as containing a few questions of a more general nature. Some questions were previously tested in a pilot qualitative study on the use of e-consultations. Consequently, the content of the interview guide was modified to include the feedback collected during the pilot study.

One moderator (AJF), who did not have any relationship with the interviewees beforehand, conducted the interviews. The moderator presented topics using the open-ended questions in the interview guide, facilitated the discussion and followed up with further questions. The interviewees could discuss their experiences freely. The moderator also sought to summarise discussions about each topic to verify interpretations of the GPs' answers. The interviews were conducted by phone due to long distances to GPs' offices. Interviews were audio recorded and transcribed verbatim in Norwegian. Quotes relevant for this paper were translated into English.

\section{Data analysis}

Data were analysed by a multidisciplinary research team consisting of three members (AJF, IMH and PZ) with a background in psychology, social science and health technology. Qualitative data collected from the interviews were analysed using the framework method. ${ }^{14}$ After transcription of the audio recordings, a sample of two interviews was randomly selected to let the research team familiarise itself with the transcripts and develop initial impressions and potential ideas for codes. Transcripts were then thoroughly read and independently analysed by each member of the research team. Interesting segments of text were underlined and notes were made in the margins of the transcripts to describe the content 
Table 1 Framework matrix

\section{Description}

\begin{tabular}{|c|c|}
\hline \multicolumn{2}{|l|}{ Use } \\
\hline Extent of use & The extent to which the service is used by the staff \\
\hline Inappropriate use & When the service is not used correctly \\
\hline Unsuitable for & Situations for which the service cannot be used \\
\hline Motivation and incentives & Factors affecting users' motivation to use the service \\
\hline \multicolumn{2}{|l|}{ Routines } \\
\hline Doctor's office & How the office is organised around the service \\
\hline General practitioners and staff & How the service is integrated into individual routines \\
\hline \multicolumn{2}{|l|}{ Advantages } \\
\hline Doctor's office & The main benefits for the staff of the doctor's office \\
\hline Technical challenges and limitations & Technical problems regarding the service functionality \\
\hline Time and efficiency & Impact of the service on the staff's productivity \\
\hline Economics & Economic impact of the service \\
\hline \multicolumn{2}{|l|}{ Other issues } \\
\hline Perceptions & Individual thoughts around the service \\
\hline Written communication & Impact on the communication with patients \\
\hline
\end{tabular}

of each passage with coding labels, as well as with more detailed information supporting the interpretation of the results. The members of the research team later met to share the coding labels, which they had assigned to the two transcripts. Each passage was analysed to discuss why it was interpreted as meaningful and how it could be useful to address the research questions. The coding labels used to describe each passage were compared with find similarities in the interpretations of the content and to resolve differences. Finally, a working analytical framework was developed around a set of codes that were explained by a short definition.

The remaining seven transcripts were then assigned to the three members of the research team and analysed using the analytical framework. New codes that were not included in the initial framework were assigned together with the already defined codes as additional impressions emerged. Regular team meetings were conducted during the process of analysing transcripts to discuss new codes, group together codes that were conceptually related, and to refine the initial analytical framework. The analytical framework was refined until no new codes were generated. The final analytical framework (table 1) consisted of fifteen codes grouped into five categories, each including a brief explanatory description of their meaning.

The final analytical framework was applied to all the transcripts by assigning appropriate codes to each meaningful passage of text. Data were then summarised in a framework matrix using Microsoft Excel. The framework matrix consisted of one column per interviewee and one row per code. A separate sheet was used for each of the four digital health services explored in this study. Data from transcripts were summarised using verbatim words and inserted into the corresponding cell in the framework matrix. The qualitative data included in the framework matrix were finally reviewed to make connections across interviewees and categories and to identify common themes as well as individual differences. ${ }^{15}$ Results were summarised and presented separately for each of the four digital health services.

\section{RESULTS}

Nine GPs from different offices (table 2) were interviewed in the period from September 2017 to November 2017. Each interview lasted from 30 to $60 \mathrm{~min}$.

\section{Electronic booking}

The amount of appointments available for electronic booking (e-booking) varied among practices. One GP office began by having all time slots open for e-bookings. However, the procedure was modified by keeping some time slots unavailable for e-booking in order to have more flexibility for patients who preferred to book in person or by phone. Other GP offices decided to restrict specific time slots to meetings or administrative work.

[...] sometimes there are time slots available for regular appointments, but not for e-bookings 
Table 2 Participant demographics

\begin{tabular}{llllll}
\hline ID & Gender & Age group (years) & Health region & $\begin{array}{l}\text { Distance to nearest } \\
\text { hospital (KM) }\end{array}$ & $\begin{array}{l}\text { Population of } \\
\text { municipality (2018) }\end{array}$ \\
\hline GP1 & Male & $60-69$ & South Eastern & 34 & $<50000$ \\
GP2 & Male & $50-59$ & South Eastern & 7 & $50-100000$ \\
GP3 & Female & $30-39$ & South Eastern & 7 & $50-100000$ \\
GP4 & Male & $60-69$ & Western & 3 & $100-200000$ \\
GP5 & Male & $40-49$ & South Eastern & 5 & $100-200000$ \\
GP6 & Male & $40-49$ & Western & 18 & $<50000$ \\
GP7 & Female & $60-69$ & Western & 1 & $200-300000$ \\
GP8 & Male & $50-59$ & Western & 10 & $200-300000$ \\
GP9 & Male & $40-49$ & South Eastern & 23 & $100-200000$ \\
\hline
\end{tabular}

One GP office had not yet adopted e-booking due to limitations in the functionality for managing different lists of patients for GPs of the same practice. The demand for e-booking was especially high among technology-oriented patients. The extent to which e-booking was available also varied among GP offices. Some GPs published all time slots 6 months in advance, while others only offered 1 month ahead. Requests for emergency appointments were generally not available through the service.

Despite different routines, GPs agreed that e-booking had obvious benefits and perceived the service as effective and timesaving. Reduced phone load was emphasised as a significant gain from several GPs. One of the GPs reported that he had performed a measurement that indicated about a quarter reduction in telephone load. Consequently, the staff had more time available for other important tasks. Reduced phone load also brought benefits for less technology-oriented patients, who prefer regular phone-booking. According to the GPs, patients considered e-booking useful and preferable to regular booking, mainly due to time savings. Patients were also less dependent on GP offices' opening hours. Patients with a tight schedule experienced increased autonomy as it was easier for them to book an appointment online that fitted with their schedule. GPs mentioned only a few disadvantages. Experience showed that e-booking was not suitable for everyone. Children, elderly, people not familiar with technology as well as some patients receiving psychiatric care were examples of patients who might require traditional booking alternatives. Moreover, GPs had less information on patients' reasons for requesting an appointment when the booking was made electronically.

GPs were generally satisfied with using e-booking for regular appointments, and were positive about the potential use for emergency appointments.

Doctors are generally sceptical about making emergency appointments available for e-booking, but we actually have a positive experience of this. The service is seldom used improperly.
The users of e-booking highlighted a number of limitations. For instance, when GPs made changes, these were not updated and visible until the next day. Another drawback was the impossibility of filtering unnecessary appointments in the same way as by phone, or modifying the required time for appointments. One GP highlighted some challenges in using same-day appointments, available for booking from 4 PM the day before.

It takes time for patients to understand this, so when looking for a time slot 2 days in advance, they can't find it. Then they try to book an appointment with another doctor, despite there being many time slots available with their own doctor. But we have always been concerned with explaining concepts and educating citizens on how to do this.

Improper use rarely occurred. When using e-booking, most patients generally booked single appointments, even if they might need a double appointment. GPs found it easier to clarify such matters by regular phone booking and missed the option of e-booking double appointments. Another challenge mentioned was the inappropriate request for vaccination.

\section{Electronic prescription renewal}

GPs adopted different procedures for handling electronic prescription (e-prescription) renewals. Some GP practices made new requests available in a common inbox that was checked daily, thus ensuring that renewals were processed even if the responsible GP was absent. One GP preferred to process all e-prescriptions by himself, while another interviewee delegated them to a medical secretary. GPs processed requests between consultations or at the end of the day, sometimes requiring overtime work.

In the way the GP scheme has been evolving, we may have ended up with more work in the evenings.

GPs mentioned a number of benefits of e-prescription, including fewer incoming calls, increased efficiency in administration resulting in freed up time for medical assessments, less pressure in the waiting room and in 
the GP office, improved dispatching priority and more precise communication.

Fewer phone calls is probably the main advantage in addition to less hassle in notifying that a prescription is ready, for instance.

Obvious advantages for patients were, according to GPs, time and money savings because they did not have to show up at the GP office to renew a prescription. However, one GP thought that it could become too easy to renew a prescription for patients who should have had a prescription review. The service could potentially involve additional work for GPs if they needed to explain patients why a renewal was refused, access their medical records and look up former use of medication, or receive many prescription requests at the same time. Another GP thought that the interface was slow.

The number of clicks and processes was somewhat easier-a bit easier than (the previously used) SMSservice. However, if you look at the doctor's work involved in managing the service in relation to attending patients, we spend more time.

The service was perceived by most GPs as best suited to renewals for chronic patients on complex medication schemes.

E-prescribing [...] provides a much more complete overview of when patients took their medicine. This makes it easier for other doctors in the system to follow up.

Some GPs believed the service could be an effective solution for 'simple' infections, frequently prescribed medications such as benzodiazepine and sporadic medications such as painkillers and allergy medications. The GPs were somewhat divided regarding the prescription of potentially addictive medication.

Ideally, addictive medications of benzodiazepine should not be renewed like this, but it depends on each situation.

However, other GPs held a different position regarding medications used for long-term treatment.

Frequently prescribed medications taken daily for chronic diseases are renewed once a year at a yearly control. There we can see [...] if there is anything to change $[\ldots]$. Renewal by electronic prescription removes the possibility for adjustment. [...] it is best suited for those medications that are easy for people to keep control of and understand when they should consult their doctor if there is something that isn't working well, such as recurrent urinary tract infections.

\section{E-consultation}

The service was only used to a limited extent by the patients. GPs reported 1-2 e-consultations per day, which occurred in the form of a dialogue between patient and GP. E-consultations were used for remote follow-up of health problems previously discussed during in-visits. Through an e-consultation, a GP could adjust a treatment, prescribe referrals or provide information. GPs could also assess, for example, signs of eczema on receiving pictures electronically.

It should be a known problem, of a medical nature and regarding a known patient

GPs were generally satisfied about how much could be done without an office visit.

Far better than I thought, depending on how well the patient describes the problem [...] Perhaps 20\%$30 \%$ of the situations require personal attendance

There were, however, cases in which e-consultations could not be used. These included newly emerged clinical problems, which could not be assessed remotely, as well as requests for sick leaves. Despite the requirements for e-consultations being well described, patients were more likely to use the service if motivated directly by their GP.

A request for e-consultation in this particular system must be replied to within 5 days. Most GPs reported internal routines according to which, in case of delays, other GPs from the same practice could handle those requests. In case of absence, a substitute was always assigned and information on leaves was updated. Routines varied among individual GPs, even if belonging to the same practice: e-consultations were processed during the time between visits, at lunch, at the end of the day or during the evening.

Some afternoon and evening work is often required. I try to answer continuously so that all requests are processed by the weekend. But if requests mount up, I can book time in my schedule

Most GPs perceived a positive impact in terms of reduced workload for both receptionists and GPs, less crowded waiting rooms and fewer urgent visits. E-consultation was seen as a simple and secure communication channel with patients, especially with those who have a chronic condition. This enabled a more efficient exchange of information, which was also documented in the electronic patient journal.

An e-consultation makes it much easier to have a good conversation around the follow-up of health problems

Another benefit that was perceived by many GPs was improved patient follow-up via e-consultations due to the higher availability of the GPs. GPs also agreed that some patients managed to express themselves more openly on health issues through e-consultations.

One patient wrote to me about issues he had never told anyone about before 
Despite the benefits of e-consultations, GPs mentioned that this service could not always replace regular visits. One limitation, for instance, was unclear communication.

Sometimes it's not possible to understand the patient

Use of e-consultations could imply additional work, especially after traditional working hours. Another limitation was that GPs could not initiate an e-consultation. In general, GPs were satisfied with the functionality of the platform, which made processing of e-consultations effective. A number of technical solutions was suggested to improve the service, such as the ability to process the electronic transmission of files and images. GPs also recommended a feature to disable the service in their absence, and the option for patients to choose between different GPs. E-consultations via videoconferencing was seen as the next step.

Perhaps in the future we could offer patients a videoconferencing service

\section{Electronic contact with the GP office (e-contact)}

Use of the service differed among GP offices. Those that had just started using the service received only a few contacts daily, while others with a longer experience answered up to 20-30 requests. The service was used to reschedule appointments, respond regarding blood tests or digital imaging, provide information about vaccines, payments or to simple treatment- related advice. There were, however, situations of inappropriate use, especially when patients used electronic contact with the GP office to address clinical questions. One reason might be related to unclear information regarding proper use. Another reason might be that, while this service was free of charge for patients, e-consultations had a fee. When such situations occurred, the office redirected the requests.

E-contact with the GP office was used about as much as e-consultations. As the service was new, no formal routines had been established yet. Offices were still struggling to define boundaries between e-contacts and e-consultations. With the purpose of making the service more efficient and standardised, patients' requests were categorised by the clinical staff.

The main advantage for the GP office consisted of a reduction in the number of visits and phone contacts, which, in return, resulted in less waiting time for patients calling the office, as well as less workload for the clinical staff.

I think it's easier to give an answer [...] It's quick, and it's done without mail or phone. Because if you call people, they will talk about more. Now you can send a short reply, so the frequency is actually increasing now. It makes it a little easier to follow up and keep a close dialogue

Interviewees indicated that the service saved time for patients by avoiding unnecessary waiting time spent on the phone. Moreover, a less busy phone line had a positive effect on patients who still needed to call the office. Another benefit was that, unlike phone contacts, e-contacts were documentable.

GPs were overall satisfied with the service and its functionalities. The only major limitation was related to uncertainties among patients on whether to use e-contact or e-consultation. In this respect, the service should be improved with more clear information.

E-contacts were considered an alternative to phone contacts. Consequently, this service had a high potential of increasing efficiency and reducing workload. Younger patients with higher computer literacy could benefit more from this service.

Someone will always use the phone instead of electronic contact, such as older people. The younger population will probably like it, and I hope they will use it more

Finally, GPs were satisfied with the economic implications. The service did not require any additional cost, but it succeeded in releasing time for the health staff. The only concern was related to situations in which GPs provided answers to clinical requests without charging the fee for an e-consultation.

\section{DISCUSSION}

\section{Summary}

Use of digital services for citizens in primary care in Norway is increasing. Use of text-based e-consultations is in the early adoption phase and therefore in limited use.

The most commonly reported advantages for the GP office include reduced phone load, increased efficiency in administration and consequently more time for medical assessments, less crowded waiting rooms and more precise communication. Clear advantages for patients are ease of use, increased flexibility, increased autonomy and time and money savings.

However, some GPs raised concern that children, the elderly, people unfamiliar with technology and some patients receiving psychiatric care were examples of patients who required traditional face-to-face alternatives. There is still some scepticism about the effects in terms of efficiency and clinical utility for e-consultations.

\section{Comparison with existing literature}

GPs' perceptions towards e-booking and e-prescription renewal were almost entirely positive. Suggestions on how to improve the services included, for example, filtering of unnecessary bookings and the option to e-book double appointments. Some GPs noted that the e-contact with the GP office was sometimes confused with e-consultations by the patients. However, this tendency was not overwhelming. E-consultation was the only service entirely intended for clinical use. While the administrative services were implemented to a larger extent, the use of e-consultations by patients was still limited. Notably, some GPs expressed surprise that the clinical utility 
of e-consultations was better than expected. While it is demonstrated that written consultations between primary and specialist health have several benefits, ${ }^{716}$ less is known about how text-based communication between GPs and patients affects clinical practice.

The experiences from early large-scale implementation in Sweden indicated that the use of digital services might increase over time after they are made available. ${ }^{17}$ In this study, a common impression among the GPs was that the initial volume of use for text-based e-consultations appeared to be limited. They also appeared to have little impact on demand for physical consultations, as the available appointments for physical consultations were still fully booked. Another study has pointed out that written communication between GP and patient can both supplement and replace physical consultations. ${ }^{18}$ In this study, however, an out-of-pocket fee was not charged for using the service, making a direct comparison with the service investigated in this study questionable. The qualitative scope of this study is not suited to detect non-obvious changes in demand and, consequently, cannot rule out the possibility that e-consultations affected physical consultations. Because of limited use from the patients, the need to implement new routines in the clinic in order to handle e-consultations were modest, and the requests were often processed between physical visits, at lunch or outside regular office hours. It is likely that an increase in use of the service would require the implementation of more rigorous routines in the GPs' offices, such as by allocating a set portion of the office hours to handling electronic consultations. A recent study highlighted that GPs can be reluctant to implement alternatives to face-toface consultations, despite policy pressure. ${ }^{19}$ We did not observe this reluctance in this study, possibly due to the characteristics of the GPs included in the study, which are pointed out in the 'Strengths and limitations' section.

\section{Implications for research and/or practice}

There were different routines among GP offices and even among individual GPs from the same practice. Organisational practices have a direct impact on the use of the service by GPs, as well as on their perception of the effects. More experience is needed to standardise routines.

More defined and standardised routines, as well as more evidence of the effects, are necessary for large-scale adoption of digital health services for citizens in primary care.

Acknowledgements We would like to thank the general practitioners sharing their valuable experiences as early adopters of the services.

Contributors AJF conducted the data collection. The authors AJF, IMH and $\mathrm{PZ}$ contributed equally in the analysis of the results and the preparation of the manuscript.

Funding This study was funded by the Norwegian Centre for E-health Research as part of the project 'Nytteeffektene av digitale helsetjenester'.

Competing interests None declared.
Patient consent for publication Not required.

Ethics approval According to the Norwegian Act on Medical and Health Research $\S 2$ and $\S 4$, the study did not require approval from the regional ethics committee (REK), but the procedure for handling the data was approved by the Data Protection Officer of the University Hospital of North Norway.

Provenance and peer review Not commissioned; externally peer reviewed.

Data sharing statement The interview guide for this study is available in Norwegian and can be shared upon request.

Open access This is an open access article distributed in accordance with the Creative Commons Attribution Non Commercial (CC BY-NC 4.0) license, which permits others to distribute, remix, adapt, build upon this work non-commercially, and license their derivative works on different terms, provided the original work is properly cited, appropriate credit is given, any changes made indicated, and the use is non-commercial. See: http://creativecommons.org/licenses/by-nc/4.0/.

\section{REFERENCES}

1. Norwegian Directorate of Helath. Norway and Health: an introduction. 2011.

2. SSB, Statistics Norway. Inforation technology use in households. 2016. https://www.ssb.no/teknologi-og-innovasjon/statistikker/ ikthus/aar/2016-09-06

3. Olayiwola JN, Anderson D, Jepeal N, et al. Electronic consultations to improve the primary care-specialty care interface for cardiology in the medically underserved: a cluster-randomized controlled trial. Ann Fam Med 2016;14:133-40.

4. Pecina JL, North F. Early e-consultation face-to-face conversions. J Telemed Telecare 2016;22:269-76.

5. Ferguson T. Digital doctoring-opportunities and challenges in electronic patient-physician communication. JAMA 1998;280:1361-2.

6. Miller EA, West DM. Where's the revolution? Digital technology and health care in the internet age. J Health Polit Policy Law 2009;34:261-84.

7. Keely E, Liddy C, Afkham A. Utilization, benefits, and impact of an e-consultation service across diverse specialties and primary care providers. Telemed J E Health 2013;19:733-8.

8. Liddy C, Maranger J, Afkham A, et al. Ten steps to establishing an e-consultation service to improve access to specialist care. Telemed $J$ E Health 2013;19:982-90.

9. North F, Uthke LD, Tulledge-Scheitel SM. Internal e-consultations in an integrated multispecialty practice: a retrospective review of use, content, and outcomes. J Telemed Telecare 2015;21:151-9.

10. Tran C, Liddy C, Pinto N, et al. Impact of question content on e-consultation outcomes. Telemed J E Health 2016;22:216-22.

11. Himmelstein DU, Woolhandler S. Hope and hype: predicting the impact of electronic medical records. Health Aff 2005;24:1121-3.

12. Ludwick DA, Doucette J. Adopting electronic medical records in primary care: lessons learned from health information systems implementation experience in seven countries. Int J Med Inform 2009;78:22-31

13. Saunders B, Sim J, Kingstone T, et al. Saturation in qualitative research: exploring its conceptualization and operationalization. Qual Quant 2018;52:1893-907.

14. Gale NK, Heath G, Cameron E, et al. Using the framework method for the analysis of qualitative data in multi-disciplinary health research. BMC Med Res Methodol 2013;13:117.

15. Braun V, Clarke V. Using thematic analysis in psychology. Qual Res Psychol 2006;3:77-101.

16. Vimalananda VG, Gupte G, Seraj SM, et al. Electronic consultations (e-consults) to improve access to specialty care: a systematic review and narrative synthesis. J Telemed Telecare 2015;21:323-30.

17. Umefjord G, Sandström H, Malker H, et al. Medical text-based consultations on the Internet: a 4-year study. Int J Med Inform 2008;77:114-21.

18. Kummervold PE, Trondsen M, Andreassen $\mathrm{H}$, et al. [Patientphysician interaction over the internet]. Tidsskr Nor Laegeforen 2004;124:2633-6.

19. Brant $\mathrm{H}$, Atherton $\mathrm{H}$, Ziebland $\mathrm{S}$, et al. Using alternatives to face-toface consultations: a survey of prevalence and attitudes in general practice. Br J Gen Pract 2016;66:e460-e466. 